\title{
Influência da temperatura sobre a agregação do solo avaliada por dois métodos
}

\author{
Influence of temperature on soil aggregation assessed by two methods
}

\author{
Heliab Bomfim Nunesi, Eiyti Kato"I, Marcos Aurélio Carolino de SáIII, Vandayse \\ Abades Rosa ${ }^{\mathrm{IV}}$, Aline dos Santos de Carvalhov ${ }^{\mathrm{v}}$, Joaquim Pedro Soares Neto ${ }^{\mathrm{VI}}$
}

\begin{abstract}
Resumo
As queimadas elevam a temperatura da superfície do solo podendo reduzir sua agregação. Sendo assim objetivou-se avaliar a influência da temperatura sobre a agregação do solo em diferentes manejos pelo método de peneiramento em água e a seco. O experimento foi conduzido no laboratório de Física dos Solos da Universidade do Estado da Bahia (UNEB). O solo caracterizado como Latossolo Vermelho Amarelo foi coletado em uma área de plantio de soja em sistema de plantio direto e em uma área de cerrado preservado (Cerradão), vizinha à área cultivada com soja. O delineamento experimental foi inteiramente casualizado em esquema fatorial $2 \times 2 \times 6$, com quatro repetições, sendo o primeiro fator o manejo do solo (sistema plantio direto de soja e cerrado preservado), o segundo fator o método (peneiramento em água e a seco) e o terceiro fator a variação das temperaturas $\left(27^{\circ} \mathrm{C}, 150^{\circ} \mathrm{C}, 250^{\circ} \mathrm{C}, 350^{\circ} \mathrm{C}, 450^{\circ} \mathrm{C}, 550^{\circ} \mathrm{C}\right)$ aplicada ao solo. As variáveis analisadas foram o percentual das classes de agregados $>2 \mathrm{~mm} ; 2-1 \mathrm{~mm}, 1-0,5 \mathrm{~mm} ; 0,5-0,250 \mathrm{~mm} ; 0,250-0,106 \mathrm{~mm}$ e menores que $0,106 \mathrm{~mm}$. Também foram avaliados o diâmetro médio ponderado (DMP) e o diâmetro médio geométrico (DMG) de agregados. A exposição dos solos a altas temperaturas reduziu a sua agregação e essa redução foi mais perceptível pelo método de peneiramento a seco. O monocultivo da soja, mesmo em sistema plantio direto, reduziu a estabilidade dos agregados quando comparado à vegetação de cerrado preservado.
\end{abstract}

Palavras-chave: Queimadas; Agregados; Manejo; Conservação do solo

\begin{abstract}
The burnings raise the soil surface temperature and may reduce its aggregation. The objective of this study was to evaluate the influence of soil temperature on its aggregation from different soil management by water and dry sieving method. The experiment was conducted at the soil physics laboratory of the State University of Bahia (UNEB). The soil characterized as Oxisol was collected in a soybean plantation area under a notillage system and in a preserved cerrado area (Cerradão), close to the soybean area. The experimental design was completely randomized in a $2 \times 2 \times 6$ factorial scheme, with four replications. The first factor was soil management (no-tillage soybean and preserved cerrado), the second factor was the method (water and dry sieving) and the third factor was the temperature range $\left(27^{\circ} \mathrm{C}, 150^{\circ} \mathrm{C}, 250^{\circ} \mathrm{C}, 350^{\circ} \mathrm{C}, 450^{\circ} \mathrm{C}, 550^{\circ} \mathrm{C}\right)$. The variables analyzed were the percentage of aggregate for classes $>2 \mathrm{~mm} ; 2-1 \mathrm{~mm}, 1-0,5 \mathrm{~mm} ; 0.5-0,250 \mathrm{~mm}$; $0,250-0,106 \mathrm{~mm}$ and less than 0,106 $\mathrm{mm}$. Weighted mean diameter (WMD) and geometric mean diameter (DMG) were also evaluated. The exposure of the soils to high temperatures reduced their aggregation and this reduction was more noticeable by the dry sieving method. Soil monoculture, even under no-tillage system, reduced the stability of the aggregates when compared to the preserved cerrado vegetation.
\end{abstract}

Keywords: Burned; Aggregates; Management; Soil conservation

Engenheiro Agrônomo, MSc., Programa de Pós-Graduação em Agronomia, Universidade de Brasília, Campus Universitário Darcy Ribeiro, CEP 70910-900, Brasília (DF), Brasil. heliabnunes@hotmail.com (ORCID: 0000-0003-0575-6029)

II Engenheiro Agrônomo, Dr., Programa de Pós-Graduação em Agronomia, Universidade de Brasília, Campus Universitário Darcy Ribeiro, CEP 70910-900, Brasília (DF), Brasil. kato@unb.br (ORCID: 0000-0001-5600-7947)

III Engenheiro Agrônomo, Dr., Pesquisador da Embrapa Cerrados, BR-020, km 18, s/n, Planaltina, CEP 73310-970, Brasília (DF), Brasil. marcos.sa@ embrapa.br (ORCID: 0000-0002-3835-5136)

IV Engenheira Agrônoma, Universidade do Estado da Bahia, BR-242, KM 04, s/n, Flamengo, CEP 47802-682, Barreiras (BA), Brasil. deyseabades@ hotmail.com (ORCID: 0000-0003-4929-5711)/ aline.tsc.carvalho@gmail.com (ORCID: 0000-0002-0216-1751)

Engenheira Agrônoma, Universidade do Estado da Bahia, BR-242, KM 04, s/n, Flamengo, CEP 47802-682, Barreiras (BA), Brasil. deyseabades@ hotmail.com (ORCID: 0000-0003-4929-5711)/ aline.tsc.carvalho@gmail.com (ORCID: 0000-0002-0216-1751)

vi Engenheiro Agrícola, Dr., Universidade do Estado da Bahia, BR-242, KM 04, s/n, Flamengo, CEP 47802-682, Barreiras (BA), Brasil. jpsneto@uneb. br (ORCID: 0000-0003-4121-3830) 


\section{Introdução}

Os sistemas de cultivo proporcionam mudanças significativas em diversas propriedades do solo, sejam elas físicas (ZHANG et al., 2017), químicas (AGEGNEHU; AMEDE, 2017) ou biológicas (LÖBMANN et al., 2016) em relação aos sistemas em equilíbrio. Essas mudanças podem ser benéficas ou prejudiciais à qualidade do solo. Um sistema amplamente difundido e aprovado em solos das regiões tropicais é o sistema plantio direto (SPD), principalmente em solos arenosos de baixa agregação. No entanto, esse deve ser combinado a outras técnicas de conservação do solo (DIDONÉ; MINELLA; EVRARD, 2017). Esse sistema baseia-se nos princípios de rotação de culturas, cobertura e não revolvimento do solo.

Contrastando com os princípios do SPD, ainda é realizado no Brasil sistemas de cultivo que utilizam o fogo como prática de manejo para controle de plantas daninhas e pragas diversas. Nesse contexto, o efeito da temperatura sobre as propriedades do solo tem se tornado cada vez mais assunto de pesquisas em diversas áreas do conhecimento científico (BENTO-GONÇALVES et al., 2012). Estima-se que em torno de $30 \%$ da superfície terrestre sofra com queimadas sazonais de diversas intensidades e duração (CHUVIECO; GIGLIO; JUSTICE, 2008). Os principais agentes responsáveis pelo fogo são o homem e os relâmpagos, principalmente, os de corrente contínua (PINEDA; MONTANYÀ; VAN DER VELDE, 2014). O horário e o tipo de vegetação também influenciam na ocorrência e duração dos incêndios (MÜLLER; VACIK, 2017), e a cada dia técnicas mais avançadas são utilizadas no monitoramento desses fenômenos de ordem natural ou antrópica (LOPES; MACHADO, 2017).

O fogo ao aquecer a superfície do solo provoca modificações em suas propriedades químicas (ALCAÑIZ et al., 2016), físicas e biológicas (MATAIX-SOLERA et al., 2011). Chen et al. (2016) relatam que temperaturas superiores a $100^{\circ} \mathrm{C}$, a depender do tempo de duração, podem provocar redução da matéria orgânica, alterações em argilas e diminuir a capacidade de saturação do solo. A agregação do solo também pode ser afetada de formas diversas pelo aquecimento, podendo ocorrer redução na estabilidade dos agregados em solos que foram submetidos às queimadas (THOMAZ, 2011; CHEN; SHRESTHA, 2012). Alguns autores preferem estudar o efeito da temperatura sobre características do solo em laboratório, para se evitar variações que possam ocorrer no ambiente. Badía-Villas et al. (2014) observaram em experimento conduzido em laboratório que as altas temperaturas reduziram o grau de repelência do solo à água e a estabilidade de agregados, provocando modificações na matéria orgânica do solo.

Contrapondo os trabalhos citados anteriormente, alguns pesquisadores relatam um aumento significativo na estabilidade dos agregados submetidos às altas temperaturas. Thomaz (2017) observou aumento significativo na estabilidade dos agregados do solo, principalmente nos macroagregados $(>2 \mathrm{~mm}$ e $>4 \mathrm{~mm}$ ), e ressalta ainda a importância do método empregado na análise para se detectar diferenças entre os sistemas com e sem a utilização de fogo. Thomaz (2011) afirma que o método de peneiramento em água é mais eficiente em detectar o efeito do fogo sobre o solo.

Muitos são os métodos para se determinar o estado de agregação do solo e entre os mais simples e mais utilizados, destaca-se o peneiramento, que pode ser tanto a seco quanto em água. No peneiramento em água, a amostra sobre peneiras de diâmetro de malha decrescente é submetida a ciclos oscilatórios verticais em água. Já no peneiramento via seco a amostra sobre peneiras semelhantes à do método em água é agitada com agitador eletromecânico que promove trepidações de diversas intensidades (SALTON et al., 2012). Diante do discutido, objetivou-se com esse trabalho avaliar a influência da temperatura sobre a agregação do solo cultivado em sistema plantio direto e sob cerrado nativo pelo método de peneiramento em água e a seco. 


\section{Material e métodos}

\section{Condução do experimento}

O experimento foi conduzido no laboratório de Física dos Solos da Universidade do Estado da Bahia (UNEB). De acordo o Sistema Brasileiro de Classificação de Solos (EMBRAPA, 2006), o solo da área em estudo foi classificado como Latossolo Vermelho Amarelo de textura média (Tabela 1) e foi coletado em uma área de plantio de soja em sistema de plantio direto (SPD) e em uma área de cerrado preservado, vizinha à área cultivada com soja sendo que nenhuma das duas áreas sofreram com incêndios nos últimos dez anos. A área cultivada com soja foi aberta há 30 anos e se iniciou com plantio convencional com correção do solo e adubação fosfatada. Há 13 anos é cultivada com soja em sistema de plantio direto sem rotação de culturas, com pousio durante o período sem chuvas que dura de abril a setembro de cada ano.

\section{Delineamento experimental}

O delineamento experimental foi inteiramente casualizado em esquema fatorial $2 \times 2 \times 6$, com quatro repetições, totalizando 96 amostras, sendo o primeiro fator o manejo do solo (sistema plantio direto de soja e cerrado preservado), o segundo fator o método (peneiramento em água e a seco) e o terceiro fator a variação das temperaturas $\left(27^{\circ} \mathrm{C}, 150^{\circ} \mathrm{C}, 250^{\circ} \mathrm{C}, 350^{\circ} \mathrm{C}, 450^{\circ} \mathrm{C}, 550^{\circ} \mathrm{C}\right)$.

\section{Coleta e caracterização dos solos}

O solo tanto na área cultivada com soja quanto na área de cerrado preservado foi coletado na camada de $0-0,20 \mathrm{~m}$. Em cada área foram amostrados quatro pontos aleatoriamente com 20 $m$ de distância entre eles com auxílio de cilindro metálico com capacidade de $1000 \mathrm{~cm}^{3}(0,1274$ $\mathrm{m}$ de altura e $0,10 \mathrm{~m}$ de diâmetro). Para coleta das amostras preservadas, o solo foi umedecido para que houvesse a menor perturbação possível de sua estrutura. Após a infiltração completa da água os cilindros foram cravados no solo e em seguida escavados em sua lateral para serem retirados sem a perturbação das amostras. Em seguida, esses foram envoltos em filme plástico, acondicionados em latas lacradas e enviadas para o laboratório de Física dos Solos da UNEB para realização das análises.

\section{Processamento das amostras}

No laboratório, as amostras foram secas ao ar ainda dentro dos cilindros por $48 \mathrm{~h}$ para facilitar o destorroamento e retirada dos cilindros. Após esse tempo, as amostras eram cuidadosamente destorroadas, rompendo manualmente os agregados em seus pontos de fragilidade e postas para secar ao ar por $48 \mathrm{~h}$ para se realizar a separação de fragmentos de raízes e superfícies espelhadas provocadas pelo corte com o cilindro. Depois de destorroadas, passadas em peneira de malha de $2 \mathrm{~mm}$ e secas ao ar foi retirada uma alíquota da amostra para caracterização física e química (EMBRAPA, 1997) (Tabela 1). A outra parte da amostra foi passada em peneiras de malha de 10 e 8 $\mathrm{mm}$ respectivamente, sendo selecionados para análise os agregados que passaram na peneira de 10 $\mathrm{mm}$ e ficaram retidas na de $8 \mathrm{~mm}$. Dos agregados selecionados foram retiradas amostras de $50 \mathrm{~g}$ para serem submetidas aos tratamentos de variação de temperatura e 20 g para determinação da umidade gravimétrica (EMBRAPA, 1997). 
Tabela 1 - Características químicas e físicas de um Latossolo Vermelho Amarelo agricultado e sob cerrado preservado na região Oeste da Bahia, Brasil.

Table 1 - Chemical and physical characteristics of Oxisol farmed and under cerrado in the western region of Bahia state, Brazil.

\begin{tabular}{|c|c|c|c|}
\hline \multicolumn{2}{|c|}{ Análise química } & \multirow{2}{*}{$\frac{\text { Soja }}{6,7}$} & \multirow{2}{*}{$\frac{\text { Cerrado }}{4,6}$} \\
\hline $\mathbf{p H}\left(\mathbf{H}_{2} \mathbf{O}\right)$ & - & & \\
\hline $\mathrm{Ca}^{2+}+\mathrm{Mg}^{2+}$ & $\mathrm{cmol}_{\mathrm{c}} \mathrm{dm}^{-3}$ & 5,67 & 1,39 \\
\hline $\mathrm{Ca}^{2+}$ & $\mathrm{cmol}_{\mathrm{c}} \mathrm{dm}^{-3}$ & 4,00 & 0,88 \\
\hline $\mathbf{A l}^{3+}$ & $\mathrm{cmol}_{\mathrm{c}} \mathrm{dm}^{-3}$ & 0,00 & 0,54 \\
\hline $\mathbf{A l}^{3+}+\mathbf{H}^{+}$ & $\mathrm{cmol}_{\mathrm{c}} \mathrm{dm}^{-3}$ & 2,47 & 5,99 \\
\hline $\mathbf{K}^{+}$ & ppm & 143,21 & 56,00 \\
\hline $\mathbf{P}($ Mel $)$ & ppm & 37,33 & 0,85 \\
\hline C & $\%$ & 1,53 & 1,41 \\
\hline Matéria orgânica & $\%$ & 2,63 & 2,43 \\
\hline Saturação por Al & $\%$ & 0,00 & 26,16 \\
\hline \multicolumn{4}{|c|}{ Granulometria } \\
\hline Areia & $\mathrm{g} \mathrm{kg}^{-1}$ & 750,9 & 741,4 \\
\hline Silte & $\mathrm{g} \mathrm{kg}^{-1}$ & 26,1 & 27,1 \\
\hline Argila & $\mathrm{g} \mathrm{kg}^{-1}$ & 223,0 & 231,5 \\
\hline
\end{tabular}

Em que: $\mathrm{pH}=$ Potencial hidrogeniônico; $\mathrm{Ca}^{2+}=$ Cálcio; $\mathrm{Mg}^{2+}=$ Magnésio; $\mathrm{Al}^{3+}=$ Alumínio; $\mathrm{Al}^{3+}+\mathrm{H}^{+}=$Acidez potencial; $\mathrm{K}^{+}=$ Potássio; $\mathrm{P}=$ Fósforo; $\mathrm{C}=$ Carbono.

\section{Aplicação dos tratamentos e análise das amostras}

Os agregados selecionados foram transferidos para placas de Petri com $15 \mathrm{~cm}$ de diâmetro para evitar a sobreposição dos mesmos, permitindo assim que todos recebessem a mesma intensidade de energia térmica. O aquecimento foi realizado em um forno Mulfla durante $10 \mathrm{~min}$ às temperaturas de $150^{\circ} \mathrm{C}, 250^{\circ} \mathrm{C}, 350^{\circ} \mathrm{C}, 450 \mathrm{C}, 550^{\circ} \mathrm{C}$ com marcha de aquecimento de $15^{\circ} \mathrm{C}$ por minuto e uma amostra mantida à temperatura ambiente $\left(25^{\circ} \mathrm{C}\right)$ foi usada como testemunha. Após aplicação dos tratamentos, as amostras ficaram em repouso por $24 \mathrm{~h}$ para a avaliação da estabilidade dos agregados por peneiramento em água e a seco.

\section{Peneiramento em água e a seco}

As amostras com $50 \mathrm{~g}$ de solo, após serem aquecidas, foram transferidas para um conjunto de peneiras do aparelho de Yoder e saturadas lentamente durante $10 \mathrm{~min}$ com auxílio de um atomizador com água deionizada. Após a saturação, as amostras sobre a peneira de $2 \mathrm{~mm}$ sobreposta às peneiras de $1 \mathrm{~mm} ; 0,5 \mathrm{~mm} ; 0,250 \mathrm{~mm}$ e 0,106 $\mathrm{mm}$ foram submersas em lâmina de água de $3 \mathrm{~cm}$ de altura e tamisadas verticalmente por 15 min para separação das classes de agregados. O aparelho utilizado 
oscilava na frequência de 30 ciclos por minuto com curso de $3 \mathrm{~cm}$. Esse era composto por quatro conjuntos de peneiras de 0,145 $\mathrm{m}$ de diâmetro e 0,04 $\mathrm{m}$ de altura. Transcorrido os 15 minutos os agregados retidos em cada peneira foram transferidos para cápsula de alumínio com auxílio de um jato de água desferido com um frasco pisseta. Essas foram levadas a estufa de circulação de ar a $105^{\circ} \mathrm{C}$ por $24 \mathrm{~h}$ para posterior pesagem visando à determinação da massa seca de cada classe de agregados.

Para o peneiramento a seco, as amostras foram transferidas para um conjunto de peneiras com as mesmas malhas descritas no procedimento anterior. As amostras foram processadas por 15 minutos e em seguida retiraram-se os agregados retidos em cada peneira para pesagem.

\section{Variáveis analisadas e análise estatística}

As variáveis analisadas foram o percentual das classes de agregados $>2 \mathrm{~mm}$; 2-1 mm, 1-0,5 $\mathrm{mm} ; 0,5-0,250 \mathrm{~mm} ; 0,250-0,106 \mathrm{~mm}$ e menores que 0,106 $\mathrm{mm}$. Também foram avaliados o diâmetro médio ponderado (DMP) e o diâmetro médio geométrico (DMG) de acordo as equações descritas em Kemper e Rosenau (1986):

$$
D M P=\sum_{i=1}^{n}(x i . w i)
$$

Em que: $x i$ = diâmetro médio das classes de agregados em milímetros; wi = proporção de cada classe em relação ao total.

$$
D M G=\left(\exp \sum_{i=1}^{n}(w p \cdot \log x i)\right) /\left(\sum_{i=1}^{n} w i\right)
$$

Em que: $w p$ = peso dos agregados de cada classe, em gramas; $x i$ = diâmetro médio das classes de agregados, em milímetros; wi = proporção de cada classe de agregados em relação ao total.

Visando atender os pressupostos da análise de variância (ANOVA), os dados foram testados quanto à normalidade para se realizar a análise. As fontes de variação significativas pelo teste $\mathrm{F}$ a $5 \%$ de probabilidade de erro foram submetidas ao teste de agrupamento de médias de Scott-Knott a 5\% de probabilidade de erro, com auxílio do programa estatístico ASSISTAT 7.7 pt. Foram ajustadas regressões para a variável temperatura.

\section{Resultados e discussão}

$\mathrm{O}$ teste $\mathrm{F}$ da ANOVA indicou interação entre todos os fatores $(\mathrm{p}<0,01 \mathrm{e}$ coeficiente de variação de 7,46\%), logo, para os fatores qualitativos (manejo do solo e método de peneiramento) foi aplicado o teste de agrupamento de dados de Scott-Knott $(p<0,05)$ e para as variáveis quantitativas (variação de temperatura) foram ajustadas regressões.

O percentual de agregados maiores que $2 \mathrm{~mm}$ no solo sob cerrado apresentou as maiores médias tanto para o método de peneiramento em água quanto para o método de peneiramento a seco (Tabela 2), o que era esperado pois a vegetação perene proporciona maior agregação quando comparadas com culturas anuais (CHRENKOVÁ et al., 2014). O método de peneiramento a seco se mostrou mais sensível em indicar diferenças entre os sistemas, visto que a diferença entre os sistemas para esse método foi de $47,86 \%$, enquanto no método de peneiramento em água foi de $17,28 \%$ o que difere de Thomaz (2011) que afirma que o método de peneiramento em água é mais eficiente em detectar o efeito do fogo sobre a agregação do solo. Provavelmente, o método via seca tenha apresentado maiores diferenças pelo fato de temperaturas elevadas provocarem acréscimo no grau de repelência dos agregados à água (BADÍAVILLAS et al., 2014), tornando esses menos suscetíveis à desagregação pela água.

As demais classes de agregados até $0,25 \mathrm{~mm}$ tiveram o mesmo comportamento que os agregados maiores que $2 \mathrm{~mm}$ no método de peneiramento a seco. No entanto, no método de peneiramento em 
água, as demais classes de agregados foram sempre superiores no solo cultivado, com exceção dos agregados menores que $0,106 \mathrm{~mm}$ que não se diferenciaram com a utilização desse método. Para o método de peneiramento a seco, todas as classes inferiores a $0,25 \mathrm{~mm}$ foram superiores no solo sob cerrado, devido à quebra maior dos agregados $>2 \mathrm{~mm}$.

\section{Tabela 2 - Distribuição das classes de agregados em função do manejo do solo (Cerrado e sistema plantio direto de soja) e do método utilizado (Peneiramento em água e peneiramento a seco).}

Table 2 - Distribution of the classes of aggregates as a function of soil management (Cerrado and monoculture of soybean) and the method used (Water screening and dry sieving).

\begin{tabular}{|c|c|c|}
\hline & \multicolumn{2}{|c|}{$\%>2 \mathrm{~mm}$} \\
\hline Manejo & Pen. em Água & Pen. a Seco \\
\hline Cerrado & 90,71 aA & $14,04 \mathrm{aB}$ \\
\hline \multirow[t]{2}{*}{ Soja } & $75,03 \mathrm{bA}$ & $7,32 \mathrm{bB}$ \\
\hline & \multicolumn{2}{|c|}{ 2-1 mm } \\
\hline Manejo & Pen. em Água & Pen. a Seco \\
\hline Cerrado & $0,99 \mathrm{bB}$ & $2,78 \mathrm{aA}$ \\
\hline \multirow[t]{2}{*}{ Soja } & 4,85 aA & $1,09 \mathrm{bB}$ \\
\hline & \multicolumn{2}{|c|}{$1-0,5 \mathrm{~mm}$} \\
\hline Manejo & Pen. em Água & Pen. a Seco \\
\hline Cerrado & $1,32 \mathrm{bB}$ & 15,56 aA \\
\hline \multirow[t]{2}{*}{ Soja } & $6,81 \mathrm{aB}$ & $8,72 \mathrm{bA}$ \\
\hline & \multicolumn{2}{|c|}{$0,5-0,25 \mathrm{~mm}$} \\
\hline Manejo & Pen. em Água & Pen. a Seco \\
\hline Cerrado & $1,94 \mathrm{bB}$ & $25,96 \mathrm{bA}$ \\
\hline \multirow[t]{2}{*}{ Soja } & $6,76 \mathrm{aB}$ & $27,42 \mathrm{aA}$ \\
\hline & \multicolumn{2}{|c|}{$0,25-0,106 \mathrm{~mm}$} \\
\hline Manejo & Pen. em Água & Pen. a Seco \\
\hline Cerrado & $1,51 \mathrm{aB}$ & $34,03 \mathrm{bA}$ \\
\hline \multirow[t]{2}{*}{ Soja } & $3,44 \mathrm{aB}$ & $42,02 \mathrm{aA}$ \\
\hline & \multicolumn{2}{|c|}{$<0,106 \mathrm{~mm}$} \\
\hline Manejo & Pen. em Água & Pen. a Seco \\
\hline Cerrado & $3,49 \mathrm{aB}$ & $7,59 \mathrm{bA}$ \\
\hline Soja & $3,07 \mathrm{aB}$ & $13,49 \mathrm{aA}$ \\
\hline
\end{tabular}

Médias seguidas pela mesma letra em cada classe de agregado, minúscula na coluna e maiúsculas na linha, não diferem pelo teste $\mathrm{F}$ a $5 \%$ de probabilidade de erro.

Na Figura 1 estão apresentadas as tendências da variável agregados maiores que $2 \mathrm{~mm}$ em função da temperatura para o solo sob cerrado e cultivado com soja. Os solos apresentaram 
comportamentos distintos, sendo que, para o solo de cerrado, a temperatura provocou redução no percentual de agregados maiores que $2 \mathrm{~mm}$. Já para o solo cultivado, a temperatura elevou esses percentuais até certo ponto (Figura 1). Thomaz (2017) também relata acréscimo significativo na estabilidade dos agregados em solos submetidos a queimadas, principalmente na classe maior que 2 e $4 \mathrm{~mm}$. Alcañiz et al. (2016) observaram alterações nas propriedades químicas do solo em áreas imediatamente após queimadas, com elevação significativa dos níveis de $\mathrm{Ca}^{2+}$. Bronick e Lal (2005) em revisão sobre estrutura do solo citam que agregados contendo $\mathrm{Ca}^{2+}$ são mais resistentes à hidratação, o que pode justificar o acréscimo na estabilidade dos agregados para o solo cultivado.

Normalmente solos sob vegetação perene tem maior atividade biológica e os agregados são estabilizados por compostos orgânicos excretados por sua fauna. Esses são chamados de biogênicos, são mais estáveis em água e com maior teor de carbono (SILVA NETO et al., 2010). Chen et al. (2016) relatam que temperaturas superiores a $100^{\circ} \mathrm{C}$ a depender do tempo de duração já podem provocar redução da matéria orgânica e alterações em argilas. Sendo assim por predominar na área de cerrado agregados biogênicos esses podem ter sido mais prejudicados pela temperatura que os agregados da área de soja onde predominam agregados pedogênicos. Segundo Campo et al. (2014) solos em que a matéria orgânica é o principal agente estabilizador dos agregados, são muito propensos à desagregação em função da temperatura.

Figura 1 - Percentual de agregados maiores que $2 \mathrm{~mm}$ em função da variação da temperatura de aquecimento em forno Mulfla em dois solos $(+=$ Solo sob cerrado preservado; $\mathbf{X}=$ Solo cultivado com soja), $\mathrm{t}=$ Temperatura; AgrCE = Agregados em solo sob cerrado; AgrSJ = Agregados em solo cultivado com soja; ${ }^{*}=$ Significativo $(\mathbf{p}<\mathbf{0 , 0 5})$. As regressões foram feitas com os dados médios dos agregados a seco e em água.

Figure 1 -Percentage of aggregates larger than $2 \mathrm{~mm}$ as a function of the variation of the heating temperature in a Mulfla oven in two soils $(+=$ Soil under preserved cerrado, $\mathrm{X}=$ Soil cultivated with soybean), $\mathrm{t}=$ Temperature; AgrCE = Aggregates in soil under cerrado; AgrSJ = Aggregates in soil cultivated with soybean; ${ }^{*}=$ Significant $(\mathrm{p}<0,05)$. The regressions were done with the average data of the aggregates dry and in water.

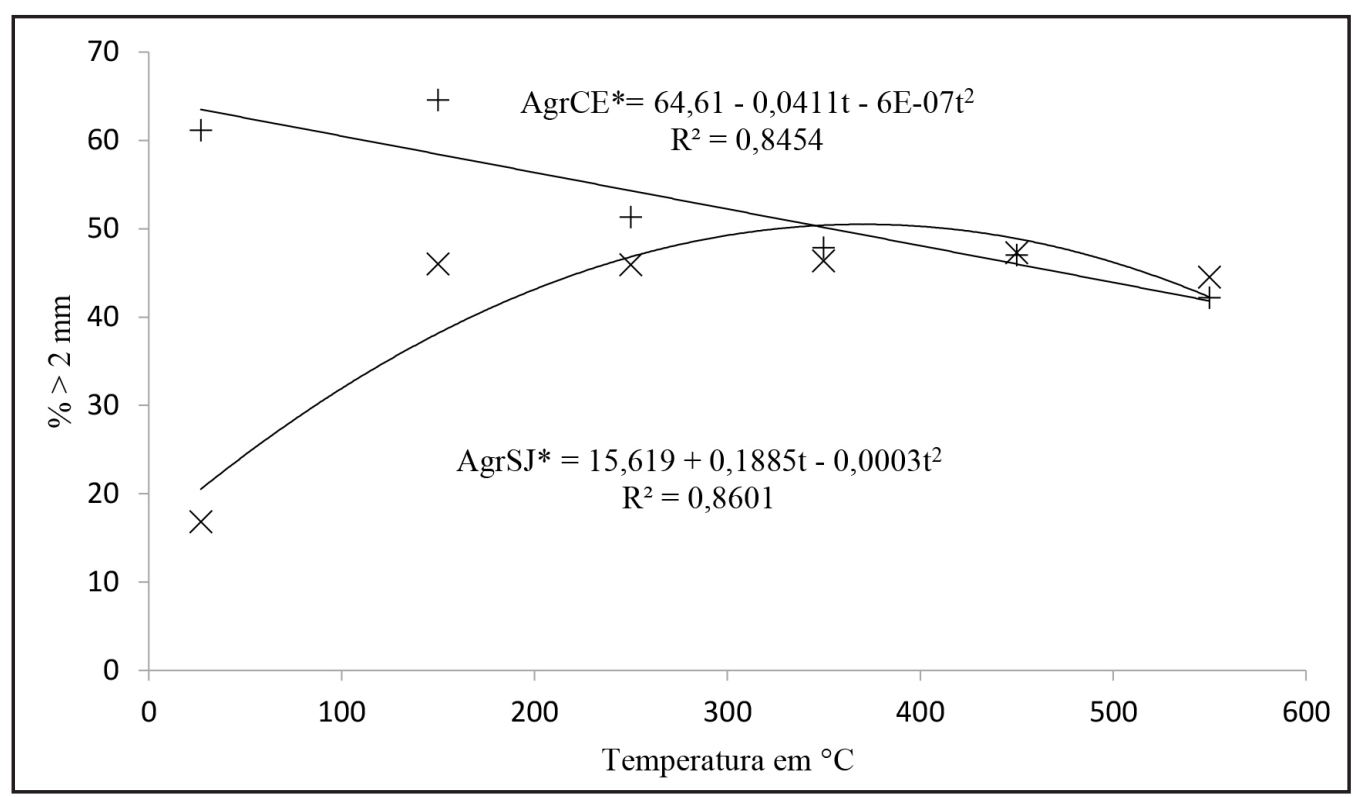

Fonte: Autores (2018)

Observa-se na Figura 2 que o método de peneiramento em água indicou acréscimo, até certo ponto, dos agregados maiores que $2 \mathrm{~mm}$ com a elevação da temperatura, tendo o seu 
ponto de máxima na temperatura de $417,83^{\circ} \mathrm{C}$. Já o método de peneiramento a seco, indicou sempre o decréscimo da quantidade de agregados com a elevação da temperatura, sendo esse mais indicado para se detectar interferências da temperatura sobre a estabilidade dos agregados do solo. Provavelmente, a menor desagregação no método de peneiramento em água está relacionada com o grau de repelência à água que se eleva com a elevação da temperatura (CAMPO et al., 2014).

A estabilidade em água dos agregados maiores que $2 \mathrm{~mm}$ nos solos estudados pode estar relacionada com os teores de $\mathrm{CaCO}_{3}$, visto que esse reduz em temperaturas próximas de $500^{\circ} \mathrm{C}$ (MATAIX-SOLERA et al., 2011), e a redução dos agregados no peneiramento em água iniciou-se na temperatura de $417,83^{\circ} \mathrm{C}$.

Figura 2 - Percentual de agregados maiores que $2 \mathrm{~mm}$ em função da variação da temperatura de aquecimento em forno Mulfla e do método empregado ( $+=$ Peneiramento em água; $\mathbf{X}=$ Peneiramento a seco), $\mathrm{t}=$ Temperatura; AgrPA = Agregados peneirados em água; AgrPS = Agregados Peneirados a seco; ${ }^{*}=$ Significativo $(\mathbf{p}<0,05)$.

Figure 2 - Percentage of aggregates larger than $2 \mathrm{~mm}$ as a function of the variation of the heating temperature in the Mulfla furnace and the method used $(+=$ Water screening, $X=$ Dry screening), $\mathrm{t}=$ Temperature; AgrPA = Aggregates sifted in water; AgrPS = Aggregates Dry sieved;

${ }^{*}=$ Significant $(\mathrm{p}<0.05)$.

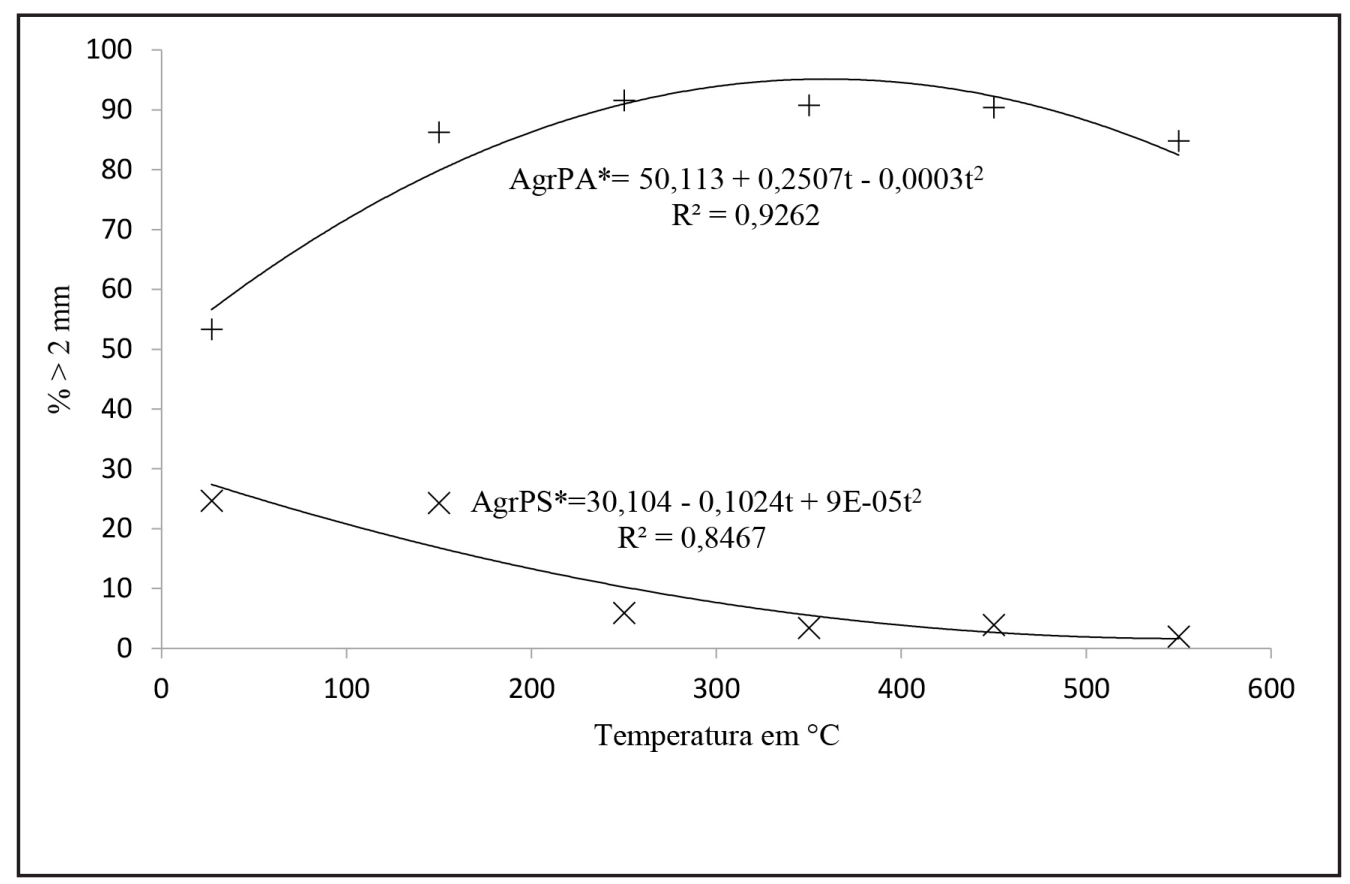

Fonte: Autores (2018)

Observa-se na Tabela 3 que o solo sob cerrado foi superior para as variáveis diâmetro médio ponderado (DMP) e diâmetro médio geométrico (DMG) independentemente do método utilizado, e que o método de peneiramento a seco detectou diferenças mais expressivas da ação da temperatura sobre a agregação do solo. 
Tabela 3 - Diâmetro médio ponderado (DMP) e diâmetro médio geométrico (DMG) em função do manejo do solo (Cerrado e sistema plantio direto de soja) e do método utilizado (Peneiramento em água e peneiramento a seco).

Table 3 - Weighted mean diameter (WMD) and geometric mean diameter (DMG) as a function of soil management (Cerrado and monoculture of soybean) and the method used (Water screening and dry sieving).

\begin{tabular}{lcc}
\hline & \multicolumn{2}{c}{ DMP } \\
\hline Manejo & Pen. em Água & Pen. a Seco \\
\cline { 2 - 3 } Cerrado & $8,20 \mathrm{aA}$ & $1,57 \mathrm{aB}$ \\
Soja & $6,90 \mathrm{bA}$ & $0,90 \mathrm{bB}$ \\
\hline & \multicolumn{2}{c}{ DMG } \\
\hline Manejo & Pen. em Água & Pen. a Seco \\
Cerrado & $6,45 \mathrm{aA}$ & $0,48 \mathrm{aB}$ \\
Soja & $5,22 \mathrm{bA}$ & $0,29 \mathrm{aB}$ \\
\hline
\end{tabular}

Médias seguidas pela mesma letra minúscula na coluna e maiúsculas na linha não diferem pelo teste $\mathrm{F}$ a $5 \%$ de probabilidade de erro.

Figura 3 - Diâmetro médio ponderado em função da variação da temperatura de aquecimento em forno Mulfla em dois solos (+ = Solo sob cerrado preservado; $X$ = Solo cultivado com soja), $t$ $=$ Temperatura; DMPCE $=$ Diâmetro médio ponderado em solo sob cerrado; DMPSJ $=$ Diâmetro médio ponderado em solo cultivado com soja; ${ }^{*}=$ Significativo $(\mathbf{p}<0,05)$.

Figure 3 - Weighted average diameter as a function of the variation of the heating temperature in a Mulfla oven in two soils $(+=$ Soil under preserved cerrado, $X=$ Soil cultivated with soybean), $\mathrm{t}=$ Temperature; DMPCE $=$ weighted average diameter in closed soil; DMPSJ = Weighted mean diameter in soil cultivated with soybean; ${ }^{*}=$ Significant $(\mathrm{p}<0.05)$.

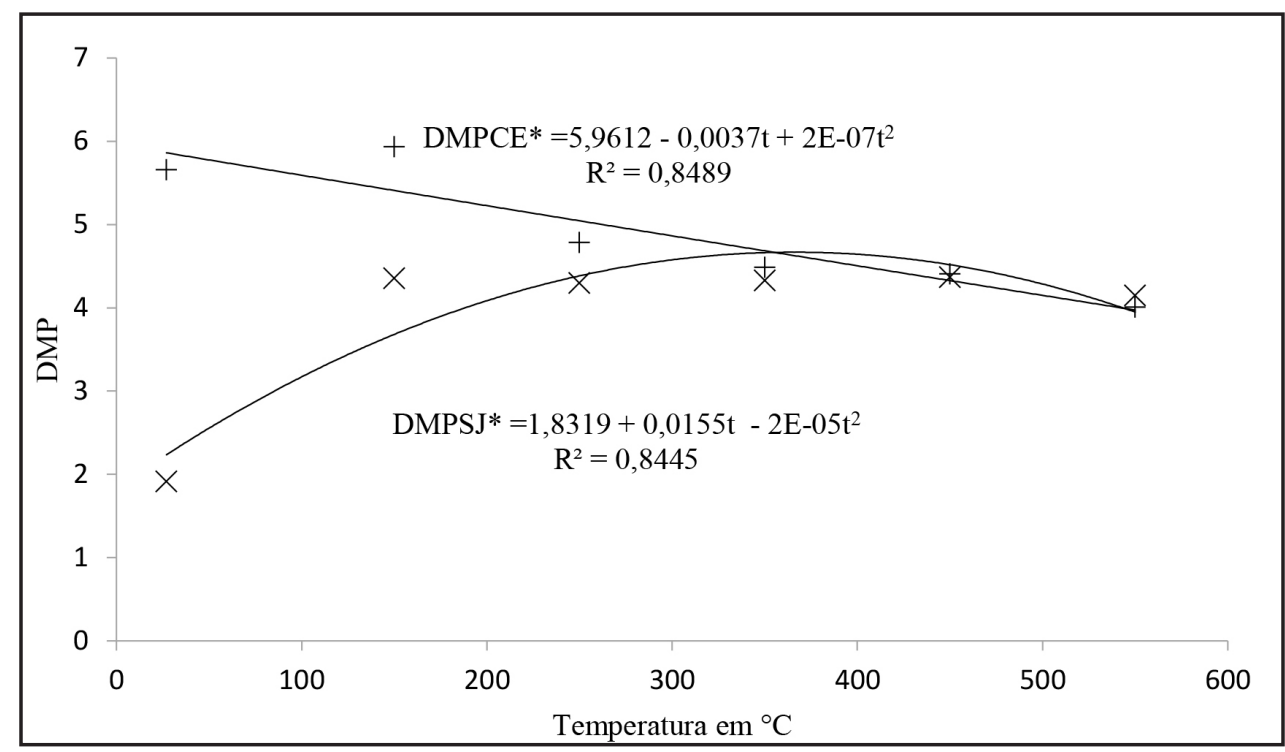

Fonte: Autores (2018) 
Figura 4 - Diâmetro médio geométrico em função da variação da temperatura de aquecimento em forno Mulfla em dois solos (+ = Solo sob cerrado preservado; $\mathbf{X}=$ Solo cultivado com soja), $\mathbf{t}=$ Temperatura; DMGCE = Diâmetro médio geométrico em solo sob cerrado; DMGSJ = Diâmetro médio geométrico em solo cultivado com soja; * ${ }^{*}$ Significativo $(\mathbf{p}<0,05)$.

Figure 4 - Geometric mean diameter as a function of the variation of the heating temperature in a Mulfla oven in two soils $(+=$ Soil under preserved cerrado, $\mathrm{X}=$ Soil cultivated with soybean), $\mathrm{t}=$ Temperature; $\mathrm{DMGCE}=$ Geometric mean diameter in closed soil; DMGSJ = Geometric mean diameter in soil cultivated with soybean; ${ }^{*}=\operatorname{Significant}(\mathrm{p}<0.05)$.

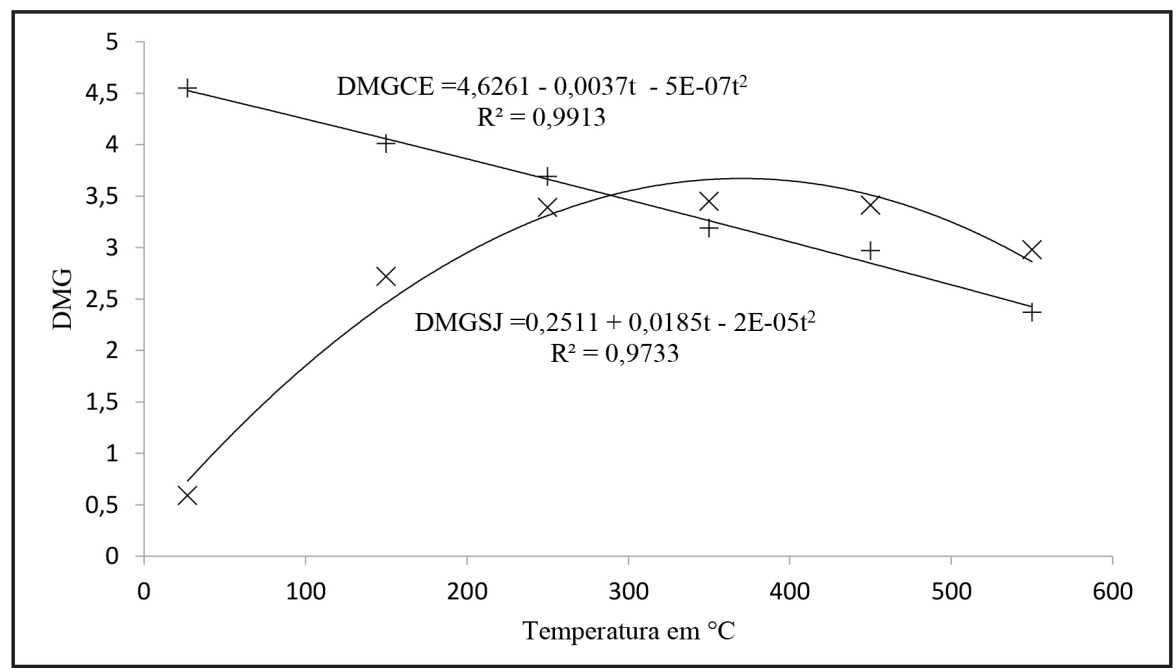

Fonte: Autores (2018)

Figura 5 - Diâmetro médio ponderado em função da variação da temperatura de aquecimento em forno Mulfla e do método empregado (+ = Peneiramento em água; $\mathbf{X}=$ Peneiramento $\mathbf{a}$ seco), $\mathbf{t}=$ Temperatura; DMPPA = Diâmetro médio ponderado pelo método de peneiramento em água; DMPPA

$=$ Diâmetro médio ponderado pelo método de peneiramento a seco; ${ }^{*}=$ Significativo $(\mathbf{p}<0,05)$.

Figure 5 - Weighted average diameter as a function of the variation of the heating temperature in the Mulfla furnace and the method used $(+=$ Water screening, $\mathrm{X}=$ Dry screening), $\mathrm{t}=$ Temperature; DMPPA = weighted average diameter by the screening method in water; DMPPA = Weighted average diameter by the dry screening method; ${ }^{*}=$ Significant $(\mathrm{p}<0.05)$.

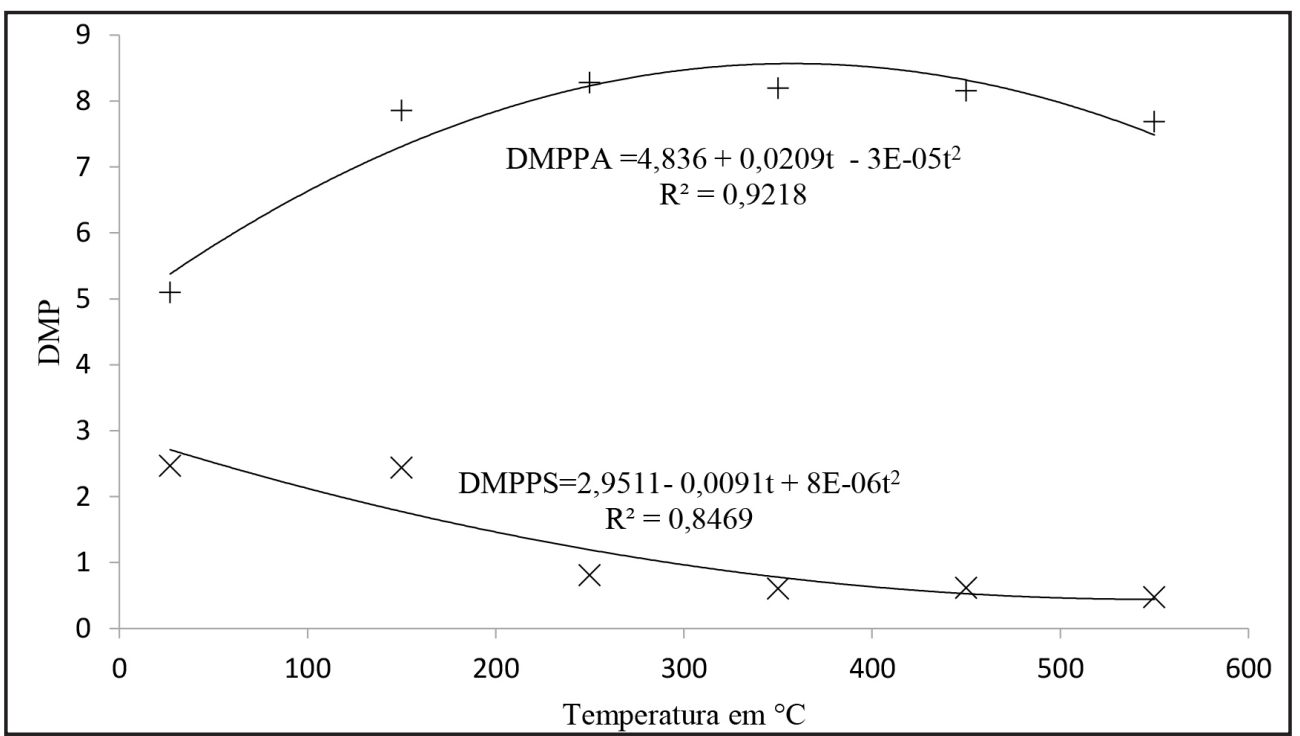

Fonte: Autores (2018) 
Figura 6 - Diâmetro médio geométrico em função da variação da temperatura de aquecimento em forno Mulfla e do método empregado (+ = Peneiramento em água; $X=$ Peneiramento a seco), DMGPA= Diâmetro médio geométrico pelo método de peneiramento em água; $\mathrm{DMGPA}=$ Diâmetro médio geométrico pelo método de peneiramento a seco; ${ }^{*}=$ Significativo $(\mathbf{p}<0,05)$.

Figure 6 - Geometric mean diameter as a function of the variation of the heating temperature in the Mulfla furnace and the method employed ( $+=$ water screening, $\mathrm{X}=$ dry screening), DMGPA = geometric mean diameter by the water screening method; DMGPA = Geometric mean diameter by the dry sieving method; ${ }^{*}=$ Significant $(\mathrm{p}<0.05)$.

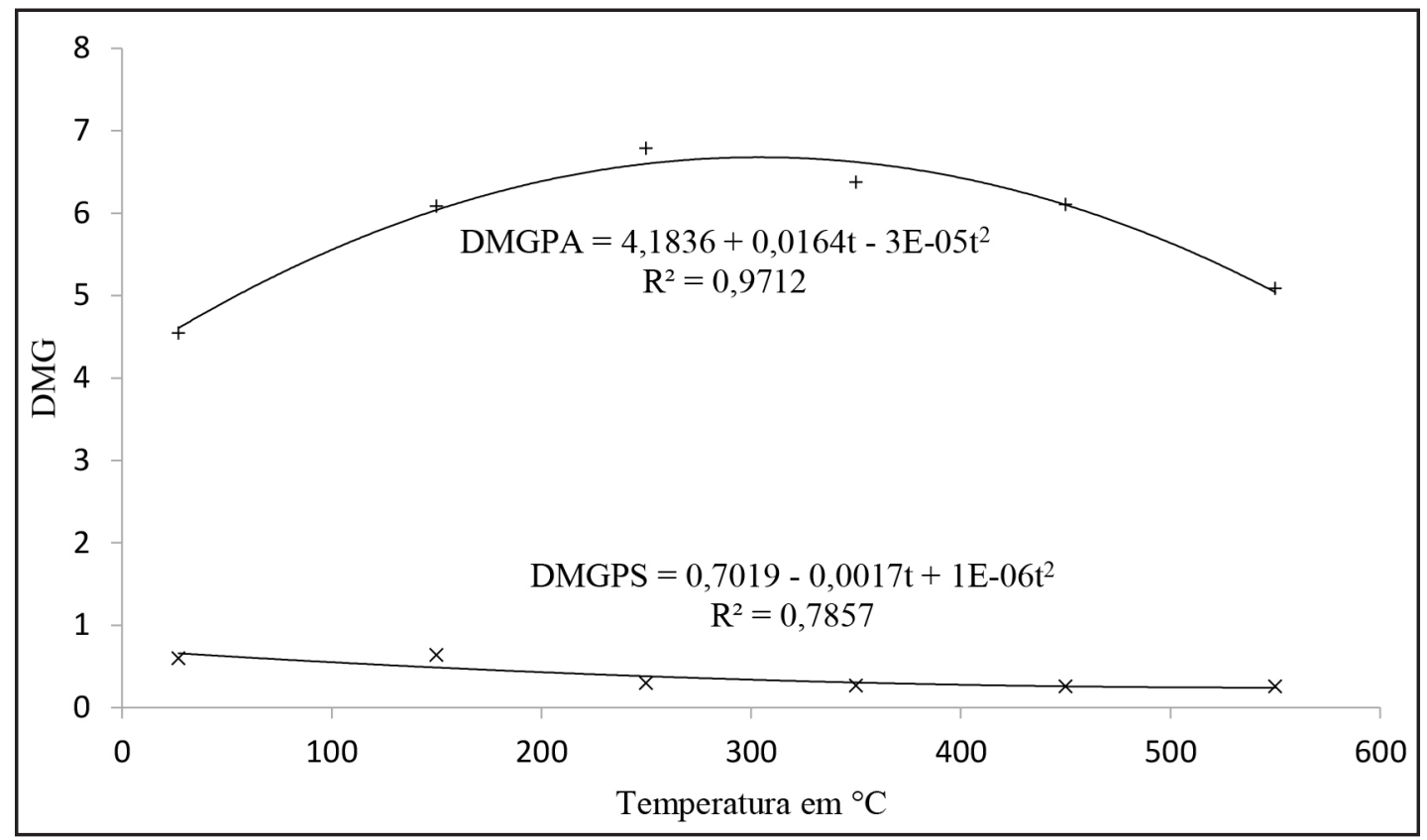

Fonte: Autores (2018)

Com relação ao método, tanto o DMP quanto o DMG tiveram o mesmo comportamento (Figuras 5 e 6). O método de peneiramento em água indica acréscimo nessas variáveis até determinada temperatura e em seguida redução. O DMP foi crescente até $348,33^{\circ} \mathrm{C}$ e o DMG até $273,33^{\circ} \mathrm{C}$.

\section{Conclusões}

A exposição dos solos a altas temperaturas reduziu a sua agregação e essa redução foi mais perceptível pelo método de peneiramento a seco.

Os métodos diferem quanto à eficiência em distinguir o impacto da temperatura sobre a agregação do solo, logo, em solos onde foram realizadas queimadas, o método de peneiramento a seco é mais sensível para detectar diferenças.

O monocultivo da soja, mesmo em sistema plantio direto, reduziu a estabilidade dos agregados quando comparado à vegetação de cerrado preservado.

\section{Referências}

AGEGNEHU, G.; AMEDE, T. Integrated soil fertility and plant nutrient management in tropical agro-ecosystems: a review. Pedosphere, Nanjing, v. 27, n. 4, p. 662-680, 2017.

ALCAÑIZ, M. et al. Long-term dynamics of soil chemical properties after a prescribed fire in a Mediterranean forest (Montgrí Massif, Catalonia, Spain). Science of the Total Environment, Barcelona, v. 572, p. 1329-1335, 2016. 
BADÍA-VILLAS, D. et al. Changes in water repellency, aggregation and organic matter of a mollic horizon burned in laboratory: soil depth affected by fire. Geoderma, Pequim, v. 213, p. 400-407, 2014.

BENTO-GONÇALVES, A. et al. Fire and soils: key concepts and recent advances. Geoderma, Pequim, v. 191, p. 3-13, 2012.

BRONICK, C. J.; LAL, R. Soil structure and management: a review. Geoderma, Pequim, v. 124, n. 1, p. 3-22, 2005.

CAMPO, J. et al. Cementing agents involved in the macro-and microaggregation of a Mediterranean shrubland soil under laboratory heating. Catena, Amsterdam, v. 113, p. 165-176, 2014.

CHEN, H. Y. H.; SHRESTHA, B. M. Stand age, fire and clearcutting affect soil organic carbon and aggregation of mineral soils in boreal forests. Soil Biology and Biochemistry, Leicestershire, v. 50, p. $149-157,2012$.

CHEN, Z. et al. Experimental study on physical properties of soft soil after high temperature exposure. Engineering Geology, Clemson, v. 204, p. 14-22, 2016.

CHRENKOVÁ, K. et al. Long-term changes in soil aggregation comparing forest and agricultural land use in different Mediterranean soil types. Geoderma, Pequim, v. 235, p. 290-299, 2014.

CHUVIECO, E.; GIGLIO, L.; JUSTICE, C. Global characterization of fire activity: toward defining fire regimes from Earth observation data. Global Change Biology, Urbana-Champaign, v. 14, n. 7, p. 1488-1502, 2008.

DIDONÉ, E. J.; MINELLA, J. P. G.; EVRARD, O. Measuring and modelling soil erosion and sediment yields in a large cultivated catchment under no-till of Southern Brazil. Soil and Tillage Research, Santa Rosa, v. 174, p. 24-33, 2017.

EMBRAPA. Centro Nacional de Pesquisa de Solos. Sistema brasileiro de classificação de solos. Rio de Janeiro, 2006. 306 p.

EMBRAPA. Manual de métodos de análise de solo. Rio de Janeiro: EMBRAPA Solos, 1997.

KEMPER, W. D.; ROSENAU, R. C. Aggregate stability and size distribution. Madison: [s. n.], 1986.

LÖBMANN, M. T. et al. The occurrence of pathogen suppressive soils in Sweden in relation to soil biota, soil properties, and farming practices. Applied Soil Ecology, Firenze, v. 107, p. 57-65, 2016.

LOPES, A. M.; MACHADO, J. A. T. Computational comparison and pattern visualization of forest fires. Chaos, Solitons \& Fractals, [s. l.], v. 102, p. 407-413, sept. 2017.

MATAIX-SOLERA, J. et al. Fire effects on soil aggregation: a review. Earth-Science Reviews, Roma, v. 109, n. 1, p. 44-60, 2011.

MÜLLER, M. M.; VACIK, H. Characteristics of lightnings igniting forest fires in Austria. Agricultural and Forest Meteorology, New Haven, v. 240, p. 26-34, 2017.

PINEDA, N.; MONTANYÀ, J.; VAN DER VELDE, O. A. Characteristics of lightning related to wildfire ignitions in Catalonia. Atmospheric research, León, v. 135, p. 380-387, 2014.

SALTON, J. C. et al. Determinação da agregação do solo-metodologia em uso na Embrapa Agropecuária Oeste. [S. l.]: EMBRAP̧A, 2012. (Comunicado Técnico).

SILVA NETO, L. F. et al. Atributos físicos e químicos de agregados pedogênicos e de coprólitos de minhocas em diferentes classes de solos da Paraíba. Ciência e agrotecnologia, Lavras, v. 34, n. 6, p. 1365-1371, nov./dez. 2010.

THOMAZ, E. L. Fire changes the larger aggregate size classes in slash-and-burn agricultural systems. Soil and Tillage Research, Santa Rosa, v. 165, p. 210-217, 2017.

THOMAZ, E. L. Influência da temperatura no diâmetro e na estabilidade de agregados em chernossolo, Saskatchewan, Canadá. Ciencia del Suelo, Santa Fe, v. 29, n. 2, p. 277-284, 2011.

ZHANG, X. et al. Effects of tillage and residue managements on organic C accumulation and soil aggregation in a sandy loam soil of the North China Plain. Catena, Amsterdam, v. 156, p. 176-183, 2017. 